\title{
Myeloerythroid-restricted progenitors are sufficient to confer radioprotection and provide the majority of day $8 \mathrm{CFU}-\mathrm{S}$

\author{
Thanyaphong Na Nakorn, ${ }^{1}$ David Traver, ${ }^{1}$ Irving L. Weissman, ${ }^{1}$ and Koichi Akashi ${ }^{2}$ \\ ${ }^{1}$ Department of Pathology and Department of Developmental Biology, Stanford University School of Medicine, \\ Stanford, California, USA \\ ${ }^{2}$ Department of Cancer Immunology and AIDS, Dana-Farber Cancer Institute, Boston, Massachusetts, USA
}

Address correspondence to: Thanyaphong Na Nakorn, Department of Pathology, 279 Campus Drive, Beckman Center, Room B261, Stanford University School of Medicine, Stanford, California 94305, USA. Phone: (650) 725-5808; Fax: (650) 498-6255; E-mail: nanakorn@leland.stanford.edu.

David Traver's present address is: Children's Hospital, Harvard University, Boston, Massachusetts, USA.

Thanyaphong Na Nakorn and David Traver contributed equally to this work.

Received for publication February 13, 2002, and accepted in revised form May 8, 2002.

\begin{abstract}
Whole-body irradiation at the minimal lethal dose causes bone marrow failure and death within 12-18 days. To identify the principal components of the hematopoietic system that are radioprotective, we transplanted lethally irradiated mice with purified progenitors: common myeloid progenitors (CMPs), megakaryocyte/erythrocyte-restricted progenitors (MEPs), or granulocyte/monocyte-restricted progenitors (GMPs). Transplanted CMPs gave rise to cells both of the granulocyte/monocyte (GM) series and the megakaryocyte/erythrocyte series, whereas GMPs or MEPs showed reconstitution of only GM or ME cells, respectively. CMPs and MEPs but not GMPs protected mice in a dose-dependent manner, suggesting that erythrocytes, platelets, or both are the critical effectors of radioprotection. Accordingly, CMPs and MEPs formed robust colonies in recipient bone marrow and spleen, whereas GMPs formed small colonies that rapidly disappeared. Direct comparisons of spleen CFU (CFU-S) potentials among each progenitor subset showed that MEPs contain the vast majority of day 8 CFU-S activity, suggesting that day 8 CFU-S are the precursors of radioprotective cell subsets. All animals radioprotected for 30 days subsequently survived for at least 6 months post-transplant, and showed only hostderived hematopoiesis after 30 days. These findings suggest that rare hematopoietic stem cells survive myeloablation that can eventually repopulate irradiated hosts if myeloerythroid-restricted progenitors transiently rescue ablated animals through the critical window of bone marrow failure.
\end{abstract}

J. Clin. Invest. 109:1579-1585 (2002). doi:10.1172/JCI200215272.

\section{Introduction}

Hematopoietic stem cells (HSCs) are clonogenic, selfrenewing multipotent progenitors (MPPs) that generate all blood cell types to maintain hematopoiesis. Using assays for clonal precursors of myeloerythroid cells, B cells, and T cells, clonogenic mouse HSCs and MPPs were phenotypically defined and prospectively isolated $(1,2)$. These cells represent about one of every 2,000 bone marrow cells in young adult C57BL/KaThy1.1 mice, and are about 2,000-fold enriched in the ability to radioprotect lethally irradiated congenic or syngeneic hosts $(1,2)$. Normally, lethally irradiated mice receiving no transplanted cells die of hematopoietic failure at 12-18 days. Mice protected by HSC transplants produce myeloerythroid cells but scant lymphoid cells by this time (3). Although these findings implicate myeloerythroid elements in radioprotection, it is not clear which myeloerythroid elements are responsible. Here we determine the in vivo activities of defined lineage-committed progenitors and test their roles in radioprotection.
The initial events in HSC differentiation include the transition of highly self-renewing long-term HSCs into short-term (ST) HSCs that possess limited self-renewal activity $(2,4)$. ST-HSCs subsequently generate MPPs that give rise to committed progenitors of various lineages (2, 5-11). The existence of HSCs and oligopotent progenitors within the hematopoietic system was initially shown by in vivo clonogenic assays. Till and McCulloch (12) discovered that mouse bone marrow contains highly proliferative progenitors capable of giving rise to colonies of hematopoietic cells within the spleens of lethally irradiated hosts. The colonies they analyzed at day 10 after transplant were composed of myeloerythroid cells, but a fraction of these colonies, when reinjected into mice, contained cells capable of long-term multilineage reconstitution that gave rise to both myeloid and lymphoid progeny $(13,14)$. Further characterization of spleen CFU (CFU-S) revealed that CFU-S at day 12 after transplant (day 12 CFU-S) derived from primitive MPPs and that day 8 CFU-S derived from relatively late myeloerythroidcommitted progenitors (15). 
The development of in vitro clonogenic assays has defined subsets of progenitors of the myeloid lineages that appear to have restricted differentiation capacity (16). Unfractionated bone marrow was found to contain oligopotent CFUs for all myeloid lineage cells (CFUGEMM or CFU-Mix) (17, 18), for granulocytes and macrophages (CFU-GM) (19), and for megakaryocytes and erythrocytes (20). Monopotent CFUs for granulocytes, macrophages, erythrocytes (21), or megakaryocytes (22) were also found. We have recently described the prospective isolation of bone marrow progenitors that are the functional equivalents of the CFU-GEMM: common myeloid progenitors (CMPs), the CFU-GM (granulocyte/monocyte-restricted progenitors, or GMPs), and the CFU-ME (megakaryocyte/erythrocyte-restricted progenitors, or MEPs) (7). In vitro experiments testing progenitor/progeny relationships demonstrated that CMPs are clonogenic precursors of GMPs and MEPs, further substantiating the paradigm of progressive lineage restriction as HSCs differentiate into mature cell types (23).

Another kind of in vitro colony has been described that contains mainly large (blast) cells (24). Paired daughter cells derived from blast colonies frequently give rise to different combinations of myeloid progeny when split into identical conditions $(25,26)$. In similar experiments, paired progenitor assays using purified HSCs have also demonstrated such stochastic outcomes (27). These data collectively suggest that the lineages observed to derive from a single type of progenitor in vitro might not reflect the full commitment potential of each progenitor, and therefore, commitment models based on in vitro clonogenic assays may not correctly describe physiological differentiation processes in vivo. Here we determine the in vivo activities of CMPs, MEPs, and GMPs, and test their roles in radioprotection. We demonstrate that the vast majority of day $8 \mathrm{CFU}-\mathrm{S}$ are derived from MEPs, and that CMPs and MEPs but not GMPs protect mice from lethal-dose irradiation in a dose-dependent manner. These results indicate that day 8 CFU-S are comprised of erythrocyte/platelet precursors and that erythrocytes, platelets, or both are the critical effectors of radioprotection.

\section{Methods}

Mouse strains. The congenic mouse strains C57BL/KaThy1.1 (Ly5.1) and C57BL/Ka-Thy1.1-Ly5.2 were used as described (6). All animals were maintained in Stanford University's Research Animal Facility in accordance with Stanford University guidelines.

Cell staining and sorting. For myeloid progenitor experiments, bone marrow cells were stained with biotinylated antibodies specific for the following lineage markers: CD3 (KT31.1), CD4 (GK1.5), CD8 (53-6.7), B220 (6B2), Gr-1 (8C5), TER119, CD19 (1D3), IgM (R6-60.2; Pharmingen, San Diego, California, USA), Thy1.1 (19XE5), and IL-7R $\alpha$ (A7R34). Cells positive for lineage markers ( Lin $^{+}$cells) were partially removed with magnetic beads conjugated to sheep anti-rat IgG (Dynabeads M-450; Dynal AS, Oslo, Norway), and the remaining cells were stained with avidin-Cy5-phycoerythrin (PE) (Tricolor; Caltag Laboratories Inc., Burlingame, California, USA). Cells were stained with PE-conjugated anti-Fc $\gamma \mathrm{R}$ (2.4G2), FITC-conjugated CD34 (RAM34; Pharmingen), Texas red-conjugated anti-Sca-1 (E13-161-7), and allophycocyanin-conjugated (APC-conjugated) anti-c-Kit (2B8) monoclonal antibodies.

For sorting HSC and common lymphoid progenitor (CLP) populations, bone marrow cells were stained with unconjugated rat antibodies specific for Lin's (CD3, CD4, CD8, B220, Mac-1, Gr-1, and TER119). Lin ${ }^{+}$cells were partially removed with immunomagnetic beads conjugated to sheep anti-rat IgG, and the remaining cells were stained with Cy5-PE-conjugated goat anti-rat IgG polyclonal antibodies. After incubation with rat IgG (SigmaAldrich, St. Louis, Missouri, USA), cells were stained with FITC-conjugated anti-Thy-1.1 (19XE5), Texas red-conjugated anti-Sca-1, and APC-conjugated anti-c-Kit antibodies. IL-7R $\alpha$ was stained with biotinylated anti-IL-7R $\alpha$ antibody (A7R34) and was visualized with PE-conjugated streptavidin (Caltag Laboratories Inc.). All cell populations were sorted or analyzed using a highly modified triple laser (488-nm argon laser, 599-nm dye laser, and UV laser, FACS Vantage; Becton Dickinson Immunocytometry Systems, Mountain View, California, USA). Progenitors were purified by sorting and then re-sorting to obtain precise numbers of cells that were essentially pure for the indicated surface marker phenotype. In limiting dilution assays, the re-sort was performed using a carefully calibrated automatic cell deposition unit system (Becton Dickinson Immunocytometry Systems). This system deposited a specific number of purified cells onto OP9 stromal cell cultures in 96-well plates.

In vivo assays to determine differentiation potential of progenitors. For reconstitution assays, purified progenitors were injected into the retroorbital venous sinus of lethally irradiated (9.5 Gy delivered in a split dose) congenic mice (differing only at the Ly5 allele) with or without 200 host-type HSCs. $R A G 2^{-/-}$recipients received 4 Gy. Intrathymic injections were performed by directly injecting cells into thymi of mice that had been irradiated (6 Gy) as described previously (6). CFU-S assays were performed with 100-500 double-sorted progenitors per mouse as previously described (28).

Peripheral blood analysis. Peripheral blood was obtained from the tail vein of each mouse and collected into EDTA-containing tubes at the indicated time points. Complete blood counts were done using a CELL-DYN 3500 analyzer (Abbott Diagnostics, Mountain View, California, USA) calibrated for mouse blood samples. Flow cytometric analysis of granulocytes and monocytes was done as previously described (29). Donor-derived cells were distinguished from endogenous host cells by the expression of different Ly5 antigens (Ly5.1 vs. Ly5.2).

\section{Results}

Evaluation of in vivo colony-forming potentials of CMPs, MEPs, and GMPs. We first tested the activities of each myeloid progenitor subset in classical day 8 and day 12 CFU-S 
a
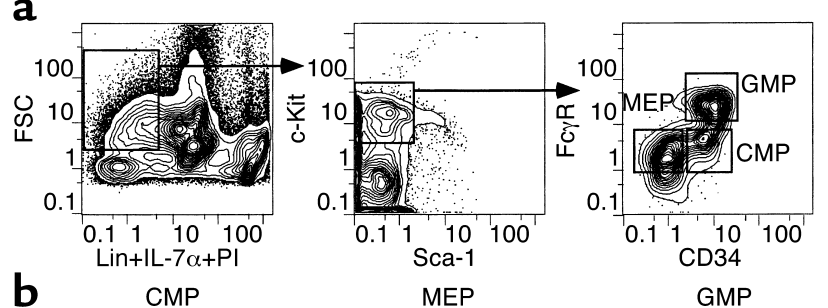

b CMP
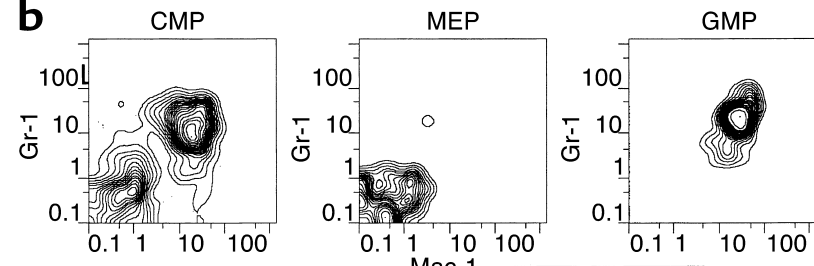
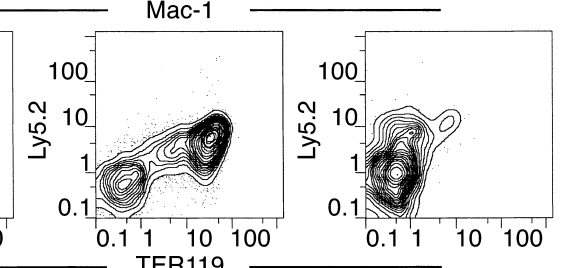

assays. Figure 1a shows the cell-surface phenotypes of CMPs, MEPs, and GMPs used in these studies. Parallel transplants comparing graded numbers of HSC and myeloid progenitor subsets showed that the majority of day 8 CFU-S activity resides within the MEP population (Table 1). The CMP population showed some day $8 \mathrm{CFU}-$ $\mathrm{S}$ activity, while GMP cells had no detectable activity. On a per cell basis, some day $12 \mathrm{CFU}-\mathrm{S}$ derived from CMPs and MEPs, but the ST-HSC/MPP subsets were most highly enriched for day 12 CFU-S activity $(1,2)$. However, when the frequencies of these cell subsets were taken into account, about half of CFU-S day 12 activity in whole bone marrow resided within MEP/CMP populations and the other half within the HSC/MPP compartment. These data suggest that the majority of day $8 \mathrm{CFU}-\mathrm{S}$ are derived from MEPs, and are consistent with our previous observation that day $9 \mathrm{CFU}-\mathrm{S}$ were highly enriched in the Thy$1.1^{\text {lo }} \mathrm{Lin}^{-} \mathrm{Sca}-1^{-}$population (1). Accordingly, histological analyses of colonies within bone marrow sections showed that MEPs gave rise to large colonies composed of

\section{Figure 1}

(a) Myeloid progenitor profiles in mouse bone marrow. Live $\mathrm{Lin}^{-}$ IL-7R $\alpha$-Thy $1.1^{-}$cells were gated and analyzed for expression of the cKit and Sca-1 surface markers. The IL-7R $\alpha^{-}$Thy 1.1-Lin-Sca-1- ${ }^{-}-\mathrm{Kit}^{+}$ fraction was subdivided into $\mathrm{Fc} \gamma \mathrm{R}^{\mathrm{lo}} \mathrm{CD} 34^{+}(\mathrm{CMP})$, Fc $\gamma \mathrm{R}^{\mathrm{lo}} \mathrm{CD} 34^{-}$ (MEP), and Fc $\gamma \mathrm{R}^{\text {hi }} \mathrm{CD} 34^{+}$(GMP) populations. (b) In vivo differentiation potential of myeloid progenitors. Splenocytes from lethally irradiated congenic recipient mice were analyzed 6 days after intravenous injection of 10,000 CMPs, MEPs, or GMPs. Top row shows Gr-1/Mac-1 profiles of donor-derived (Ly5.2) cells in recipient mice (Ly5.1). Bottom row shows Ly5.2/TER119 profiles from the Gr-1-Mac-1' fractions shown above. FSC, forward scatter.

erythroid cells occasionally containing megakaryocytes but devoid of GM cells (Figure 2c). GMPs formed small diffuse colonies in bone marrow that contained only GM cells (Figure 2d). CMPs formed robust colonies in bone marrow that were composed of either ME or GM clusters (Figure 2b, left and right close-ups, respectively). In the spleen, CMPs and MEPs gave rise to colonies with similar appearances that appeared to be composed exclusively of erythroid precursors (not shown). GMPs rarely gave rise to spleen colonies; the few apparent colonies showed only diffuse clusters of macrophages.

In vivo differentiation potentials of the three myeloid progenitor subsets. We next tested the in vivo differentiation potential of these three progenitor populations. All three subsets quickly gave rise to mature cells in vivo, their progeny being detectable by 3-4 days after transplant (not shown). Six days after the injection of 10,000 CMPs into lethally irradiated recipient mice, both $\mathrm{Gr}-1^{+} \mathrm{Mac}-1^{+}$myelomonocytic cells and TER $119^{+}$erythroid cells were detectable in the spleen (Figure 1b) and bone marrow (data not shown). In contrast, 10,000 GMPs transiently gave rise to only Gr- $1^{+} \mathrm{Mac}-1^{+}$cells, while equivalent MEP transplants transiently generated TER $119^{+}$cells but not GM cells. Thus, the lineage outcomes of each progenitor subset corresponded with those previously described in vitro (7).

Myeloerythroid progenitors do not have significant self-renewal activity. To evaluate self-renewal potential and proliferative capacity, we injected $200 \mathrm{HSCs}$ from C57BL/KaThy1.1 (Ly5.1) and 5,000 cells of each myeloid progenitor population from C57BL/Ka-Thy1.1-Ly5.2 donors into lethally irradiated C57BL/6-Ly5.1 hosts. In

Table 1

CFU-S activity of hematopoietic stem and progenitor cell populations

\begin{tabular}{lllll}
\hline LT-HSCs & ST-HSCs/MPPs & CMPs & MEPs
\end{tabular}

Day 12

CFU-S frequency

Number of CFU-S $/ 10^{6}$ bone marrow cells

Day 8

CFU-S frequency

Number of CFU-S $/ 10^{6}$ bone marrow cells

$\begin{array}{cc}1 / 61^{\mathrm{A}} & 1 / 14^{\mathrm{A}} \\ 2 & 29 \\ & \\ <1 / 100^{\mathrm{A}} & <1 / 100^{\mathrm{A}} \\ <1 & <1\end{array}$

$<1$

$1 / 200$
10
$1 / 67$
30

30
$1 / 53$

19

$1 / 15$

67
$<1 / 500$

$<1$

$<1 / 500$

$<1$

CFU-S frequencies presented represent the number of spleen colonies formed per total number of cells injected from each population. Numbers of CFU-S per $10^{6}$ bone marrow cells are calculated using frequencies of LT-HSCs, ST-HSCs/MPPs, CMPs, MEPs, and GMPs of $0.01 \%, 0.04 \%, 0.2 \%, 0.1 \%$, and $0.4 \%$ of nucleated bone marrow cells, respectively. LT, long-term; ST, short-term. ${ }^{\mathrm{A} D a t a}$ taken from Morrison and Weissman (2) presented for comparison. 


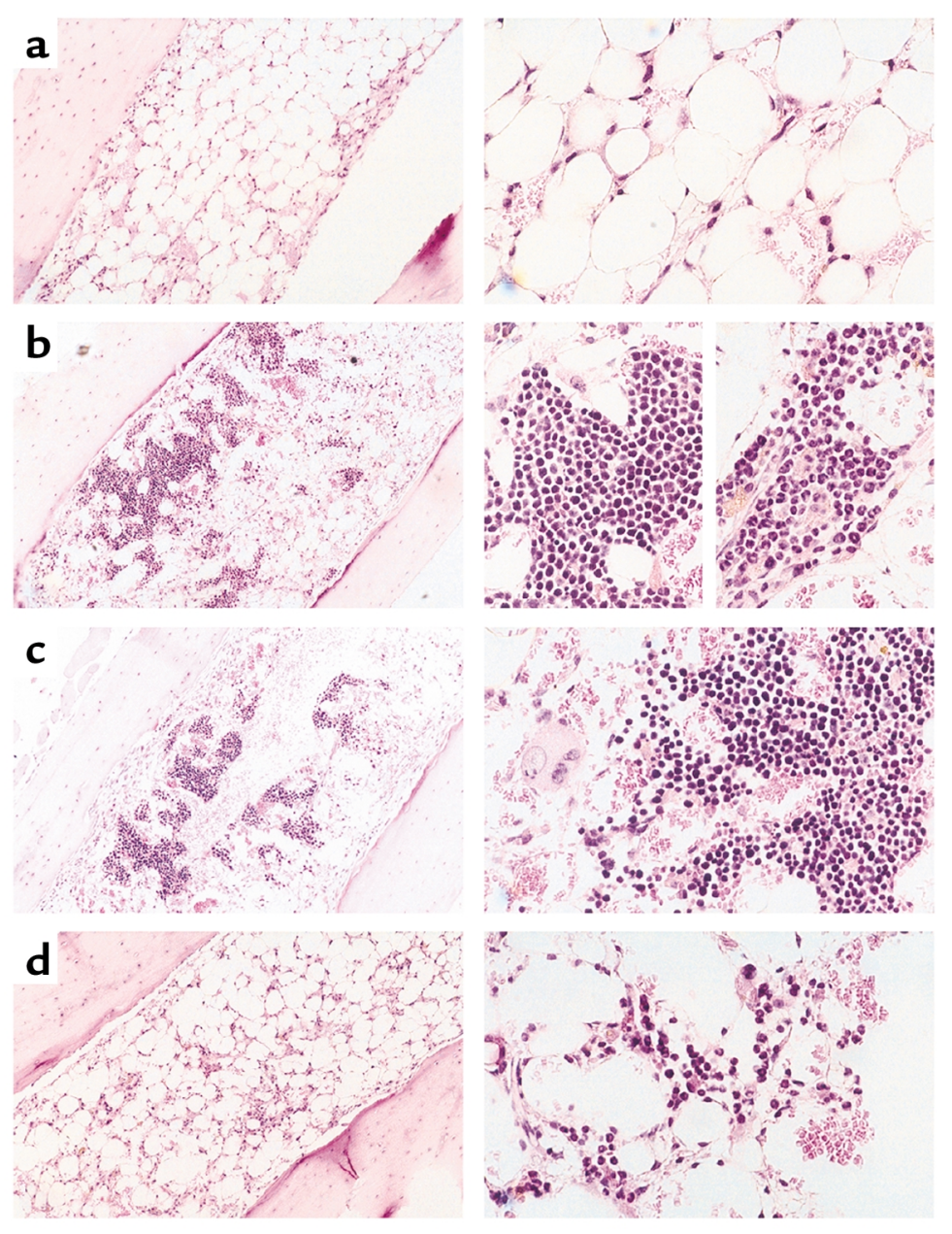

Figure 2

Morphology of colonies derived from sorted CMPs, MEPs, and GMPs. Tibias were sectioned at day 7 after transplantation of (a) no cells, (b) $1 \times 10^{4} \mathrm{CMPs},(\mathbf{c})$ $6 \times 10^{3} \mathrm{MEPs}$, and (d) $2 \times 10^{4} \mathrm{GMPs}$ into lethally irradiated recipients. Controls confirmed the absence of host-derived colonies. Left panels show field views at $\times 40$ magnification, whereas right panels are close-up views at $\times 100$ magnification. Erythroid colonies were generally larger and more compact (left close-up view in b) than were GM colonies (right close-up in b). Also note the distinct nuclear morphologies of granulocytes and monocytes in close-up colony views in $\mathbf{b}$ and $\mathbf{d}$.

we tested the capacity of these myeloidrestricted progenitors to radioprotect lethally irradiated congenic recipient mice without additional provision of HSCs or helper bone marrow. Previous experiments transplanting ST-HSCs/ MPPs whose myeloid progenies are gone by 8 weeks suggested that early radioprotection allows rare surviving host HSCs to reach radioprotective levels after the 12- to 18-day critical window of bone marrow failure (2). To exclude the possibility of contamination by HSCs, doublesorted myeloid progenitor cells (>99\% purity) were used for all transplants. While transplantation of GMPs alone provided no radioprotection, both CMP and MEP transplants showed dosedependent rescue of irradiated recipients (Figure 3, a-c). Ten thousand CMPs or

this competitive reconstitution assay, the progeny from either $\mathrm{Fc} \gamma \mathrm{R}^{\mathrm{lo}} \mathrm{CD} 34^{-}$MEP or $\mathrm{Fc} \gamma \mathrm{R}^{\mathrm{hi}} \mathrm{CD} 34^{+}$GMP cells were undetectable after 2 weeks. The myeloid progeny from $\mathrm{Fc} \gamma \mathrm{R}^{\mathrm{lo}} \mathrm{CD} 34^{+} \mathrm{CMP}$ cells were detectable at 2 weeks and 3 weeks (data not shown), but disappeared by 4 weeks after injection. This indicates that these populations have only transient repopulation potentials with limited or no self-renewal activity.

CMPs and MEPs alone provide radioprotection. Given that CMPs, GMPs, and MEPs show the same differentiation potential in vivo as previously shown in vitro,
MEPs prolonged the survival of irradiated hosts to around day 21 but failed to ensure their survival after 30 days. However, when larger numbers of CMPs or MEPs were transplanted, we began to observe longterm survivors among the recipients. Sixty percent and seventy-two percent of mice transplanted with 50,000 CMPs or MEPs, respectively, survived more than 6 weeks. Peripheral blood samples of surviving recipients were analyzed at day 42 by flow cytometry. Although all recipients showed full hematologic recovery, no donor-derived myeloid cells were present in the

Table 2

Peripheral blood cell count at day 12 after myeloid progenitor transplantation

\begin{tabular}{lcccc}
\hline & Control & CMPs & MEPs & GMPs \\
Hematocrit $(\%)$ & $24.35 \pm 2.79$ & $39.23 \pm 5.39^{\mathrm{A}}$ & $36.48 \pm 6.57^{\mathrm{A}}$ & $28.85 \pm 3.37$ \\
Number of mice with hematocrit $\geq 30 \%$ & $0 / 4$ & $4 / 4$ & $3 / 4$ & $0 / 4$ \\
Platelet count $($ per $\mu \mathrm{l})$ & $23,025 \pm 5,952$ & $46,000 \pm 13,284^{\mathrm{A}}$ & $39,975 \pm 12,451^{\mathrm{A}}$ & $29,275 \pm 6,621$ \\
Number of mice with platelets $\geq 40,000 / \mu \mathrm{l}$ & $0 / 4$ & $3 / 4$ & $2 / 4$ & $0 / 4$ \\
WBC count $($ per $\mu \mathrm{l})$ & $22.25 \pm 15.26$ & $53.75 \pm 27.43$ & $43.75 \pm 11.08$ & $37.25 \pm 15.5$ \\
Number of mice with WBCs $\geq 500 / \mu \mathrm{l}$ & $0 / 4$ & $0 / 4$ & $0 / 4$ & $0 / 4$ \\
\hline
\end{tabular}

Mice were lethally irradiated and injected with no cells (control) or 30,000 of each myeloid progenitor (CMPs, MEPs, or GMPs) at day 0. Peripheral blood was collected at day 12 and analyzed using an automated blood cell counter. Data are shown as mean \pm SEM. The critical values of blood counts are extrapolated from human clinical data. ${ }^{A} P<0.05$ compared with control. WBC, white blood cell. 


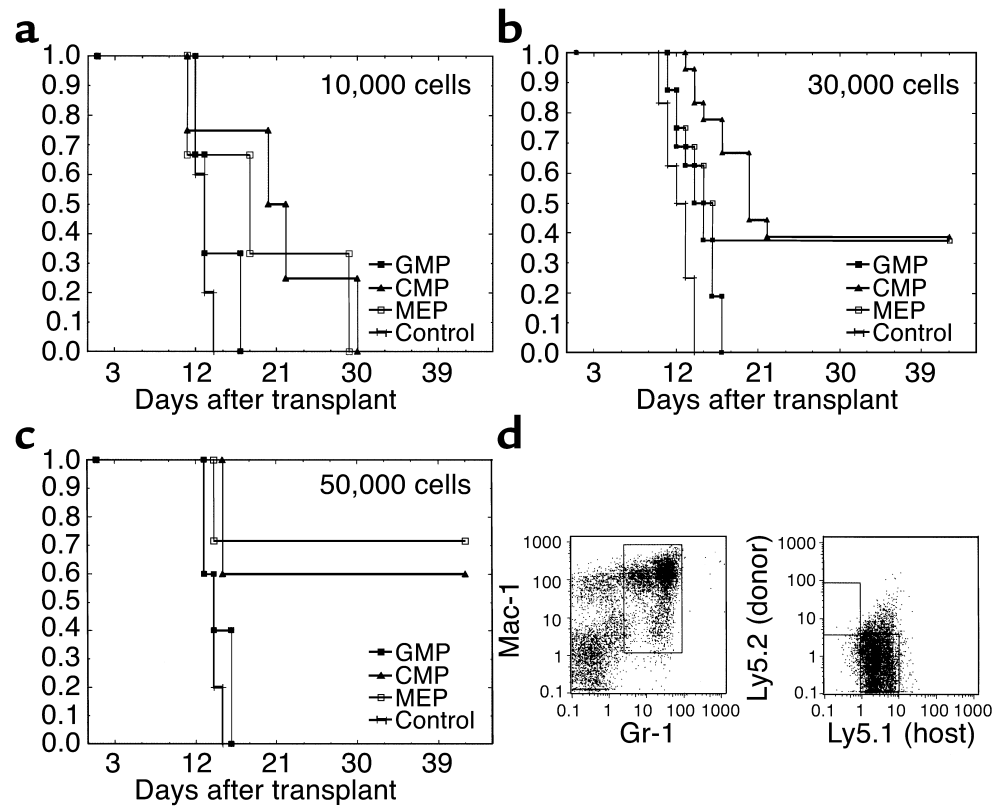

\section{Figure 3}

Radioprotection of mice transplanted with myeloid progenitors. (a-c) Kaplan-Meier survival curves of lethally irradiated mice transplanted with no cells (control, rectangles), GMPs (filled squares), MEPs (open squares), or CMPs (triangles). All mice receiving no cells died by day 14 after irradiation, consistent with hematopoietic failure. Radioprotection was defined as survival beyond day 30 . (a) No radioprotection was detected at the dose of 10,000 cells. (b) While 30,000 GMPs did not confer radioprotection, the same number of CMPs and MEPs protected $41.17 \%(7 / 17)$ and $35.29 \%(6 / 17)$ of mice from postradiation death, respectively. (c) More than $60 \%$ of mice can be saved by either 50,000 CMPs or 50,000 MEPs. (d) A representative flow cytometric analysis of peripheral blood from a recipient of 50,000 MEPs at day 42 after transplantation. All Gr-1+Mac-1+ cells are host-derived (Ly5.1).

"choice" that occurs at distinct stages downstream of HSCs. The restricted capacity in vivo of CMPs and CLPs to differentiate into myeloid or lymphoid cells also suggests that significant plas-

peripheral blood at this time point (Figure 3d), indicating that the radioprotective effect was indeed mediated by poorly self-renewing progenitor cells that do not contribute to long-term hematopoiesis.

The nadir of blood counts after the minimum lethal dose of radiation is usually at day 15 when hematocrits of less than $15 \%$ and platelet counts below $20,000 / \mu 1$ are recorded (30). To evaluate the effect of myeloid progenitors on the level of circulating blood cells, we performed complete blood counts at day 12 , before death from hematopoietic failure occurred. Mice injected with 30,000 MEPs or CMPs had significantly higher hematocrits and platelet counts than did radiation controls (Table 2). However, white blood cell counts were not significantly different between any of the groups. In fact, we never observed any recipients with more than 500 white blood cells $/ \mu \mathrm{l}$ of blood ( $10 \%$ of normal value) at this time, even when up to 100,000 CMPs or GMPs were transplanted (data not shown). Taken together, these data strongly suggested that in these studies, erythrocytes and/or platelets are the critical effectors that prevent death during this period of bone marrow failure.

\section{Discussion}

In this report, we functionally characterize three myeloerythroid-restricted progenitor subsets that have recently been isolated - CMPs and their lineal descendants, MEPs and GMPs (7). CMPs are downstream of HSCs, because CMPs do not give rise to HSCs, do not have self-renewal potential, and lack T cell differentiation potential (Figure 4). The identification of the CMPs, in addition to the previously reported CLPs (6), suggests that commitment to a myeloid or lymphoid lineage is a key developmental ticity between these lineages is rare once commitment occurs (31).

The ability to prospectively isolate the progenitors representing the major branch points of the hematopoietic tree allows the functionalities of each subset to be tested in a precise manner. In this way, we examined each stem and progenitor subset for classical in vivo colony-forming and radioprotection activity. We found that the majority of day 8 CFU-S activity resides within the MEP population and is absent in GMPs, supporting previous findings that day 8-9 CFU-S are largely erythroid $(1,15,32)$. CMPs presumably form

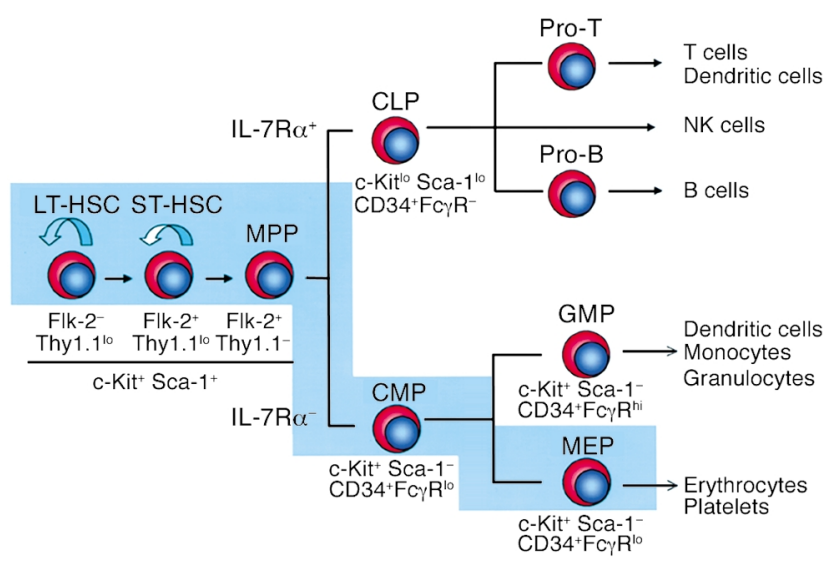

\section{Figure 4}

Proposed model of murine hematopoiesis based on prospectively isolatable bone marrow populations. HSCs are long-term self-renewing cells that generate all blood cell types. IL-7R $\alpha$ expression defines the commitment of MPPs to downstream CLPs that generate the lymphoid lineages. CMPs are clonogenic precursors of both GMPs and MEPs that respectively generate myelomonocytes and erythrocytes/platelets. Radioprotection capacity is present only in the highlighted populations that can generate erythrocytes and/or platelets in vivo. 
splenic colonies by first giving rise to MEPs. Our data also reveal that about half of day 12 CFU-S activity resides within lineage-committed progenitors and about half within the more primitive HSC/MPP population $(1,2,15)$. The day 12 activity observed within the MEP population may be explained by previous work suggesting that some day 12 colonies derive from surviving day 8 colonies (33). CLPs do not possess either day 8 or day 12 CFU-S activity (6). Thus, this study shows directly that the classical day 8 CFU-S assay largely measures MEP activity.

Radioprotection is defined as the ability to rapidly reconstitute an ablated blood-forming system in order to maintain viability of the host for a limited period of time, generally 30 days in the mouse. The populations responsible for this activity have been the subject of controversy (reviewed in ref. 34). While we have previously shown that as few as 100 HSCs can provide radioprotection $(2,3,30)$, it has been argued that HSCs do not contribute directly to radioprotection since their relative quiescence causes significant delay of effector cell production $(35,36)$. Recently, other groups have also reported that long-term HSCs have poor radioprotective capacity and require the assistance of additional bone marrow subsets for full radioprotection $(4,10)$.

We tested the capacity of myeloid-restricted CMPs, MEPs, and GMPs to radioprotect lethally irradiated congenic recipient mice without additional provision of HSCs or helper bone marrow. While transplantation of GMPs alone provided no radioprotection, both CMP and MEP transplants showed dose-dependent rescue of irradiated recipients. The fact that CMPs and MEPs but not GMPs contain radioprotective capacity, together with the results of blood cell counts at day 12 , strongly indicate that deficiencies of red blood cells, platelets, or both are normally responsible for mortality from hematopoietic failure following total body irradiation in mice. The hematocrits and platelet numbers 12 days after the injection of 30,000 MEPs or CMPs alone are comparable to the levels achieved by transplantation of a saturating dose of $\mathrm{c}-\mathrm{Kit}^{+} \mathrm{Thy}-1.1^{\mathrm{lo}} \mathrm{Lin}^{-/ \mathrm{lo}} \mathrm{Sca}-1^{+}$(KTLS) HSCs ( $\geq 5,000$ cells) (30). Interestingly, host-type cells gradually recovered in rescued recipients, and after 30 days constituted all blood cells. This is similar to transplants of radioprotective doses of ST-HSCs or MPPs (2), indicating that transplanted CMPs, MEPs, MPPs, and ST-HSCs provide transient production of erythrocytes and platelets sufficient to maintain host viability until rare, residual HSCs present in the host can take over. Therefore, in these studies, radioprotection is determined only by the capacity of cells to provide sufficient erythropoiesis and/or megakaryopoiesis during the period of bone marrow aplasia and is not the exclusive property of the HSC/MPP compartment. The requirement for high numbers of CMPs or MEPs to obtain significant radioprotection is probably due to their lack of selfrenewal capacity, relatively short life spans, and small progeny burst sizes when compared with HSCs, which can replenish the CMP and MEP pools indefinitely.
Although the spleen does not normally contribute to human hematopoiesis, there are a number of conditions in which extensive splenic hematopoiesis can occur (37). This strongly indicates that human spleens contain a microenvironment capable of supporting proliferation and differentiation of hematopoietic stem and progenitor cells in vivo. Unfortunately, very few studies have been done to examine this aspect of hematopoietic recovery after bone marrow transplantation. It is generally agreed that macroscopic spleen colonies equivalent to mouse CFU-S do not exist in humans. However, microscopic bone marrow and spleen colonies can be found in a majority of patients shortly after bone marrow transplantation $(38,39)$. Interestingly, their characteristics are very similar to what we describe here for CMP- and MEP-derived colonies. Whether these colonies originate from the human counterparts of mouse CMPs and MEPs remains to be determined.

Cytopenia after total body irradiation or high-dose chemotherapy is one of the most serious problems in clinical oncology and transplantation. In our murine model, CMPs and MEPs significantly increase hematocrit and platelet counts in ablated recipients, whereas neutropenia cannot be prevented by transplantation of myeloid-restricted progenitors. Using a mathematical model simulating the clinical situation of peripheral blood transplantation, Scheding et al. have predicted that even transplantation of $1 \times 10^{7} \mathrm{CFU}-\mathrm{GM} / \mathrm{kg}$ is not sufficient to avoid neutropenia after high-dose chemotherapy (40). We have transplanted high doses of GMPs - up to 100,000 per recipient $\left(2 \times 10^{6} / \mathrm{kg}\right)$ - and never observed significant increases in blood neutrophil counts (data not shown). However, it is likely that neutropenia alone does not cause mortality unless serious bacterial or fungal infections occur. Therefore, the importance of myeloid progenitors to providing sufficient granulopoiesis could not be clearly demonstrated in this transplantation model because all irradiated recipients were kept on antibiotics under aseptic conditions. In another experimental system, we recently found that in conjunction with HSC transplants, the addition of CMP and GMP, but not MEP populations, protects mice from lethal infection by Aspergillus or Psendomonas during this neutropenic period (A. Bitmansour et al., unpublished observations). Additionally, these progenitor populations are efficiently expanded and mobilized following standard protocols used in clinical transplantation and are all devoid of T lymphocyte differentiation potential in vivo (T. Na Nakorn, unpublished observations). Taken together, these results suggest that high-dose myeloid-restricted progenitor therapy might be effective in ameliorating the morbidity and mortality from myelosuppression caused by high-dose irradiation or chemotherapy regimens.

In summary, we have tested the behaviors of myeloerythroid-restricted progenitor subsets in a transplantation setting to model their normal physiological roles as closely as possible. All prospectively isolated myeloerythroid progenitor populations (CMPs, 
GMPs, and MEPs) showed transient reconstitution of irradiated hosts, and displayed fate outcomes in accordance with those of in vitro assays. Within the limits of the in vivo assays, they are non-self-renewing cells. CMPs and MEPs rapidly formed robust colonies in recipient marrow and spleen. Most importantly, CMPs and MEPs provided dose-dependent rescue of otherwise lethal irradiation. Our results also suggest that erythrocytes, platelets, or both are the critical effectors of radioprotection. The identification of counterpart populations in humans may allow us to test whether these progenitors can be used as adjuncts in hematopoietic cell transplantation to overcome some of the major complications seen in the clinic.

\section{Acknowledgments}

This research was supported by US Public Health Service grant CA-42551 (to I.L. Weissman) and by National Institute of Allergy and Infectious Diseases Training Grant 5 T32 AI-07290 (to D. Traver). T. Na Nakorn was supported by the Anandamahidol Foundation under the royal patronage of His Majesty the King of Thailand. K. Akashi was supported by a 1997 Jose Carreras International Leukemia Foundation Grant and the 2000 Claudia Adams-Barr program. We thank S.-I. Nishikawa for the anti-IL-7R $\alpha$ antibody, Amy Kiger for critical evaluation of the manuscript, Libuse Jerabek for excellent laboratory management and assistance with animal procedures, Veronica Braunstein for antibody preparation, the Stanford Shared FACS Facility for flow cytometer maintenance, and Lucino Hidalgo, Diosdado Escoto, and Bert Lavarro for animal care.

1. Spangrude, G.J., Heimfeld, S., and Weissman, I.L. 1988. Purification and characterization of mouse hematopoietic stem cells. Science. 241:58-62.

2. Morrison, S.J., and Weissman, I.L. 1994. The long-term repopulating subset of hematopoietic stem cells is deterministic and isolatable by phenotype. Immunity. 1:661-673.

3. Uchida, N., Aguila, H.L., Fleming, W.H., Jerabek, L., and Weissman, I.L. 1994. Rapid and sustained hematopoietic recovery in lethally irradiated mice transplanted with purified Thy- $1.1^{\mathrm{lo}} \mathrm{Lin}-\mathrm{Sca}-1^{+}$hematopoietic stem cells. Blood. 83:3758-3779.

4. Osawa, M., Hanada, K., Hamada, H., and Nakauchi, H. 1996. Long-term lymphohematopoietic reconstitution by a single CD34-low/negative hematopoietic stem cell. Science. 273:242-245.

5. Morrison, S.J., Wandycz, A.M., Hemmati, H.D., Wright, D.E., and Weissman, I.L. 1997. Identification of a lineage of multipotent hematopoietic progenitors. Development. 124:1929-1939.

6. Kondo, M., Weissman, I.L., and Akashi, K. 1997. Identification of clonogenic common lymphoid progenitors in mouse bone marrow. Cell. 91:661-672.

7. Akashi, K., Traver, D., Miyamoto, T., and Weissman, I.L. 2000. A clonogenic common myeloid progenitor that gives rise to all myeloid lineages. Nature. 404:193-197.

8. Christensen, J.L., and Weissman, I.L. 2001. Flk-2 is a marker in hematopoietic stem cell differentiation: a simple method to isolate long term stem cells. Proc. Natl. Acad. Sci. USA. 98:14541-14546.

9. Randall, T.D., Lund, F.E., Howard, M.C., and Weissman, I.L. 1996. Expression of murine CD38 defines a population of long-term reconstituting hematopoietic stem cells. Blood. 87:4057-4067.

10. Zhao, Y., et al. 2000. Murine hematopoietic stem cell characterization and its regulation in BM transplantation. Blood. 96:3016-3022.

11. Adolfsson, J., et al. 2001. Upregulation of Flt3 expression within the bone marrow Lin-Sca $1+c-k i t+$ stem cell compartment is accompanied by loss of self-renewal capacity. Immunity. 15:659-669.

12. Till, J.E., and McCulloch, E.A. 1961. A direct measure of the radiation sensitivity of normal mouse bone marrow cells. Radiat. Res. 14:213-222.
13. Siminovitch, L., McCulloch, E.A., and Till, J.E. 1963. The distribution of colony-forming cells among spleen colonies. J. Cell Comp. Physiol. 62:327-336.

14. Lepault, F., Ezine, S., and Gagnerault, M.C. 1993. T- and B-lymphocyte differentiation potentials of spleen colony-forming cells. Blood. 81:950-955.

15. Magli, M.C., Iscove, N.N., and Odartchenko, N. 1982. Transient nature of early haematopoietic spleen colonies. Nature. 295:527-529.

16. Bradley, T.R., and Metcalf, D. 1966. The growth of mouse bone marrow cells in vitro. Aust. J. Exp. Biol. Med. Sci. 44:287-299.

17. Johnson, G.R., and Metcalf, D. 1977. Pure and mixed erythroid colony formation in vitro stimulated by spleen conditioned medium with no detectable erythropoietin. Proc. Natl. Acad. Sci. USA. 74:3879-3882.

18. Metcalf, D., Johnson, G.R., and Mandel, T.E. 1979. Colony formation in agar by multipotential hemopoietic cells. J. Cell. Physiol. 98:401-420.

19. Ichikawa, Y., Pluznik, D.H., and Sachs, L. 1966. In vitro control of the development of macrophage and granulocyte colonies. Proc. Natl. Acad. Sci. USA. 56:488-495.

20. McLeod, D.L., Shreve, M.M., and Axelrad, A.A. 1976. Induction of megakaryocyte colonies with platelet formation in vitro. Nature. 261:492-494

21. Stephenson, J.R., Axelrad, A.A., McLeod, D.L., and Shreeve, M.M. 1971. Induction of colonies of hemoglobin-synthesizing cells by erythropoietin in vitro. Proc. Natl. Acad. Sci. USA. 68:1542-1546.

22. Metcalf, D., MacDonald, H.R., Odartchenko, N., and Sordat, B. 1975. Growth of mouse megakaryocyte colonies in vitro. Proc. Natl. Acad. Sci. USA. 72:1744-1748.

23. Dexter, T.M. 1990. Introduction to the haemopoietic system. Cancer Surv. 9:1-5.

24. Nakahata, T., and Ogawa, M. 1982. Identification in culture of a class of hemopoietic colony-forming units with extensive capability to self-renew and generate multipotential hemopoietic colonies. Proc. Natl. Acad. Sci. USA. 79:3843-3847.

25. Suda, J., Suda, T., and Ogawa, M. 1984. Analysis of differentiation of mouse hemopoietic stem cells in culture by sequential replating of paired progenitors. Blood. 64:393-399.

26. Suda, T., Suda, J., and Ogawa, M. 1984. Disparate differentiation in mouse hemopoietic colonies derived from paired progenitors. Proc. Natl. Acad. Sci. USA. 81:2520-2524.

27. Nakauchi, H., Takano, H., Ema, H., and Osawa, M. 1999. Further characterization of CD34-low/negative mouse hematopoietic stem cells. Ann. NY Acad. Sci. 872:57-66.

28. Traver, D., Akashi, K., Weissman, I.L., and Lagasse, E. 1998. Mice defective in two apoptosis pathways in the myeloid lineage develop acute myeloblastic leukemia. Immunity. 9:47-57.

29. Lagasse, E., and Weissman, I.L. 1996. Flow cytometric identification of murine neutrophils and monocytes. J. Immunol. Methods. 197:139-150.

30. Uchida, N., et al. 1998. High doses of purified stem cells cause early hematopoietic recovery in syngeneic and allogeneic hosts. J. Clin. Invest. 101:961-966

31. Kondo, M., et al. 2000. Cell-fate conversion of lymphoid-committed progenitors by instructive actions of cytokines. Nature. 407:383-386.

32. Humphries, R.K., Jacky, P.B., Dill, F.J., Eaves, A.C., and Eaves, C.J. 1979. CFU-S in individual erythroid colonies derived in vitro from adult mouse marrow. Nature. 279:718-720.

33. Wolf, N.S., and Priestley, G.V. 1986. Kinetics of early and late spleen colony development. Exp. Hematol. 14:676-682.

34. Morrison, S.J., Wright, D.E., Cheshier, S.H., and Weissman, I.L. 1997. Hematopoietic stem cells: challenges to expectations. Curr. Opin. Immunol. 9:216-221.

35. Jones, R.J., Wagner, J.E., Celano, P., Zicha, M.S., and Sharkis, S.J. 1990. Separation of pluripotent haematopoietic stem cells from spleen colonyforming cells. Nature. 347:188-189.

36. Jones, R.J., et al. 1996. Characterization of mouse lymphohematopoietic stem cells lacking spleen colony-forming activity. Blood. 88:487-491.

37. Lampert, I.A. 1994. Pathology of the spleen. In Disorders of the spleen. A. Cuschieri and C.D. Forbes, editors. Blackwell Scientific Publications. Oxford, United Kingdom. 51-77.

38. Cline, M.J., Gale, R.P., and Golde, D.W. 1977. Discrete clusters of hematopoietic cells in the marrow cavity of man after bone marrow transplantation. Blood. 50:709-712.

39. Antin, J.H., Weinberg, D.S., and Rappeport, J.M. 1985. Evidence that pluripotent stem cells form splenic colonies in humans after marrow transplantation. Transplantation. 39:102-105.

40. Scheding, S., et al. 1999. How many myeloid post-progenitor cells have to be transplanted to completely abrogate neutropenia after peripheral blood progenitor cell transplantation? Results of a computer simulation. Exp. Hematol. 27:956-965. 